\title{
Size-Exclusion Reaction Chromatography (SERC): A New Technique for Protein PEGylation
}

\author{
by \\ Conan J. Fee \\ Department of Materials \& Process Engineering \\ University of Waikato \\ Private Bag 3105 \\ Hamilton 2020 \\ New Zealand \\ ph: +64 78384206 \\ fax +64 78384835 \\ Email: c.fee@waikato.ac.nz
}

Keywords: PEGylation, size-exclusion, gel filtration, reaction, chromatography 


\section{Abstract}

A new, widely applicable process that combines reaction and separation in a single unit operation is described. The process, size-exclusion reaction chromatography (SERC), simultaneously allows control of the extent of reactions in which molecular size is altered and the separation of products and reactants. In SERC, a moving reaction zone is formed by injection of reactants onto a size-exclusion chromatography column. Reactants and products are partitioned differently within the mobile phase, resulting in different linear flow rates through the column. The products are therefore removed selectively from the reaction zone, minimising their residence time in the reaction zone and allowing their separation in the downstream section of the column. For reactions such as protein PEGylation, in which successive addition of PEG groups to the protein results in significant molecular size increases, SERC potentially offers a method by which a dominant final PEGylated protein size can be produced at high yield. The SERC PEGylation of two model proteins, $\alpha$-lactalbumin and $\beta$-lactoglobulin, is demonstrated and results show that simultaneous reaction and separation was obtained.

\section{Introduction}

The circulation half-lives of small therapeutic proteins $(<20 \mathrm{kDa})$ can be dramatically improved by increasing their molecular size through the covalent attachment of one or more polyethylene glycol (PEG) groups (Cunningham-Rundles et al. 1992; Francis et al. 1998; Gombotz and Pettit 2000). The attachment of PEG groups not only prevents early glomerular (kidney) filtration (Fung et al. 1997) but the water of hydration surrounding each PEG group moves constantly and is thought to slow immunological attack via steric hindrance (Abuchowski et al. 1977). Increasing the circulation half- 
life of a therapeutic protein has the dual benefits of increasing its overall efficacy and reducing the required frequency of dosage. The latter aspect can be an important factor in reducing patient discomfort and increasing product acceptability; particularly for treatments requiring repeated intravenous or sub-cutaneous administration.

Various PEGylation chemistries have been described, usually involving reactions between activated forms of PEG and the N-terminal amine and lysine groups of the protein (Fisher et al. 1995; Fung et al. 1997). In standard methods, the native protein is mixed with activated PEG for a specified time and the extent of reaction is controlled largely by the reaction temperature and $\mathrm{pH}$. Mono-PEGylated protein molecules are formed initially and residual active sites subsequently react to form diPEGylated molecules, and so on. The result is a distribution of products that differ both in the number of PEG groups attached and probably their attachment positions.

This sequence of reactions poses serious problems. For example, controlling the reaction time to maximise the production of a tri-PEGylated product results in a mixture containing six protein species: native protein, 1-, 2-, 3-, 4- and 5-PEGylated proteins; plus unreacted activated PEG and reaction end-products. Not only is there a poor product yield but also heterogeneity of this kind is unacceptable in a therapeutic protein so downstream processing is necessary to obtain a homogeneous end product. Downstream processing is difficult because the physicochemical properties of the PEGylated protein species are very similar, apart from differences in their effective molecular size, and each step required for downstream processing decreases overall yield. 
The low yields from PEGylation and the need for downstream processing represent significant costs for the PEGylated product over and above its native form. Given that the native therapeutic protein (generally a recombinant protein) must be purified to homogeneity prior to PEGylation, the overall production cost of the PEGylated protein is likely to be very high, offsetting the potential benefits of PEGylation.

In this paper, a new reaction method, size-exclusion reaction chromatography (SERC), is described, which combines control of the PEGylation reaction with simultaneous purification of the products. In size-exclusion chromatography, molecular species are partitioned within the mobile phase according to their molecular sizes and shapes and the pore size distribution of the stationary phase. Because different molecular species are contained within differing total mobile phase volumes they migrate through a packed column at different rates. SERC utilizes a moving reaction zone within a size-exclusion chromatography column to control the time of contact between reactants, to selectively remove products from the reaction zone and to selectively inhibit reactions based on molecular size.

A short pulse of activated PEG is injected onto a size-exclusion column running at a constant volumetric flow rate. A subsequent pulse of native protein will eventually catch up to the slower-moving PEG pulse, forming a moving reaction zone that exists for as long as the two reactant pulses occupy the same axial position in the column. The addition of a 5000 Da PEG group adds significantly more than 5000 Da to the effective molecular weight of the protein, as measured by size-exclusion chromatography, due to the water of hydration associated with the PEG group. For example, it is shown in this paper that the addition of a single 5000 Da PEG group to 
a $35.8 \mathrm{kDa}$ protein results in a mono-PEGylated protein with an apparent molecular weight in excess of $100 \mathrm{kDa}$ as measured by size exclusion in a column calibrated using protein standards. Thus the PEGylated product is partitioned differently from either of the two reactants. If the size-exclusion medium is chosen carefully, the PEGylated product will be partitioned into the void space and so will move relatively quickly out of the reaction zone. Additional column length downstream of the reaction zone allows separation of the reactants and products. By choosing appropriate reactant pulse volumes, reactant concentrations and volumetric flow rates, a great deal of control can be exercised over the reaction conditions and the length of the subsequent separation zone.

Results of SERC PEGylation of two model proteins, $\alpha$-lactalbumin and $\beta$ lactoglobulin, are presented in this paper. Expectations were that the large molecular weight products of PEGylation would be partitioned into the void volume of the sizeexclusion column and therefore be selectively removed from the (slower-moving) reaction zone, thus minimising multiple PEGylation reactions. Additionally, the sizeexclusion medium may also act to prevent the formation of a product larger than the pore size in which the reactants are located. It is surmised that this latter effect has far greater potential for controlling such reactions, through appropriate choice of the sizeexclusion media pore size, than differences in migration rates through the column.

\section{Materials \& Methods}

\section{Reagents}

Activated PEG reagent, mPEG succinimidyl propionate (mPEG-SPA), molecular weight $5000 \mathrm{Da}$, was purchased from Shearwater Corporation, Alabama. $\alpha$ - 
lactalbumin, (14,200 Da) and $\beta$-lactoglobulin dimer (35,800 Da) were purchased from Sigma-Aldrich Corporation, Australia. Gel filtration chromatograms of the two proteins and mPEG-SPA are shown in figure 1. A minor amount of $\alpha$-lactalbumin is evident in the $\beta$-lactoglobulin material but otherwise the two proteins are sufficiently pure for PEGylation studies.

All solutions were made up in $20 \mathrm{mM}$ Tris- $\mathrm{HCl}$ buffer at $\mathrm{pH}$ 7.5.

\section{Batch PEGylation}

Batch PEGylation was carried out with each protein individually to follow the reaction progress in the absence of the size-exclusion medium. $10 \mathrm{~mL}$ of $20 \mathrm{mg} / \mathrm{mL}$ mPEG-SPA solution was added to $10 \mathrm{~mL}$ of $20 \mathrm{mg} / \mathrm{mL}$ native protein and stirred in an open $25 \mathrm{~mL}$ beaker at room temperature. One sample (approx. $1 \mathrm{~mL}$ ) was withdrawn immediately after mixing the reactants and further samples were taken at 10-minute intervals for 1 hour. Samples were placed into vials and acidified with one drop of $1 \mathrm{~N} \mathrm{HCl}$ to stop the reaction.

\section{SERC PEGylation}

SERC PEGylation of each protein was carried out to determine the elution profile from the size-exclusion column and to compare the overall product profiles with the corresponding batch PEGylation results. A HiLoad 16/60 Superdex 75 pg sizeexclusion column (Amersham Pharmacia Biotech, Uppsala, Sweden) was connected to an AKTAexplorer 10XT liquid chromatography system (Amersham Pharmacia Biotech, Uppsala, Sweden) and equilibrated with two column volumes of $20 \mathrm{mM}$ Tris- $\mathrm{HCl}$ at $\mathrm{pH}$ 7.5. For $\alpha$-lactalbumin PEGylation a specified volume of native 
protein $(10 \mathrm{mg} / \mathrm{mL})$ was injected onto the column, followed immediately by an equal volume of mPEG-SPA (10 mg/mL), and vice versa for $\beta$-lactoglobulin PEGylation. UV absorbance was read at $280 \mathrm{~nm} .5 \mathrm{~mL}$ fractions were collected continuously from the outlet stream after an initial $40 \mathrm{~mL}$ of buffer (i.e. slightly less than the void volume of the column) had passed through the column. $1 \mathrm{~mL}$ samples of each fraction was withdrawn by pipette and placed into auto sampler vials for size-exclusion analysis. The remaining portions of those fractions that contained proteins (identified from previous runs) were pooled and a sample of the pool was taken for sizeexclusion analysis.

The distribution coefficients in the SERC column, $\mathrm{K}_{\mathrm{av}}$, were determined using standard methods (APBiotech ) to be $0.3500,0.1768$ and 0.2175 for $\alpha$-lactalbumin, $\beta$ lactoglobulin and mPEG-SPA, respectively. PEGylated proteins were assumed to be partitioned into the void volume, with a $\mathrm{K}_{\mathrm{av}}$ value of 0 . Reactant injection volumes and flow rates were then set to give estimated reaction zone residence times (i.e. the times for which the reactants overlapped in the column) by assuming that each reactant progressed through the column at a constant velocity, related to its $\mathrm{K}_{\mathrm{av}}$ value.

\section{Analysis}

$40 \mu \mathrm{L}$ samples were injected onto a Superdex 200 HR10/30 size-exclusion column (Amersham Pharmacia Biotech, Uppsala, Sweden) connected to an AKTAexplorer 10XT system (Amersham Pharmacia Biotech, Uppsala, Sweden) using an Autosampler A900, running phosphate buffered saline at $0.5 \mathrm{~mL} / \mathrm{min}$. The sizeexclusion column was first calibrated using standard protein molecular weight markers (Amersham Pharmacia Biotech, Uppsala, Sweden) in the range $1 \times 10^{4}-1 \mathrm{x}$ 
$10^{6}$ Da and using standard PEG molecular weight markers (Phenomenex) in the range $3 \times 10^{3}$ to $7.2 \times 10^{4}$ Da (figure 2). UV absorbance was read at $280 \mathrm{~nm}$ and the molecular weight profiles of samples recorded. Individual samples of $\alpha$-lactalbumin and $\beta$-lactoglobulin (dimer) had retention volumes consistent with their native molecular weights according to the protein standard curve, while mPEG-SPA had a retention volume consistent with that expected from the PEG standard curve (figure 1), corresponding to an equivalent protein molecular weight of $22 \mathrm{kDa}$.

\section{Results}

Batch PEGylation

Figures 3 and 4 show gel filtration chromatograms for the products of batch PEGylation of $\alpha$-lactalbumin and $\beta$-lactoglobulin, respectively.

In each case there is a shift in the peak areas with time, showing a reduction in the native protein peak, an initial increase and then decrease in the mono-PEGylated protein peak, and corresponding increases in the multiple-PEGylated species with time.

\section{SERC PEGylation}

Figures 5 and 6 show the elution profiles obtained from the SERC process for $\alpha$ lactalbumin and $\beta$-lactoglobulin, respectively. Gel filtration analyses of the fractions collected are given in Figures 7 and 8. These show that the larger molecular weight species were eluted early and that there was good separation of the protein species from the residual mPEG-SPA and other low molecular weight reaction products, 
especially for $\alpha$-lactalbumin PEGylation. Thus simultaneous reaction and separation was achieved.

Figures 9 and 10 show the pooled protein fractions eluted from the SERC process for $\alpha$-lactalbumin and $\beta$-lactoglobulin, respectively. Comparison of these figures with batch PEGylation (figures 3 and 4) shows that SERC PEGylation (3 hours) resulted in a higher ratio of mono-PEGylated product than the corresponding batch processes (1 hour).

\section{Discussion}

The results show that protein PEGylation can be carried out in a size-exclusion column and that reactants and products are separated in the process. Size-exclusion is a logical choice for the purification of PEGylated proteins and this combination of reaction and separation in a single unit operation should reduce capital costs and eliminate the need for handling and conditioning between reaction and separation steps.

Of interest in figures 3 and 4, are the peaks seen at a retention volume of approximately $20 \mathrm{~mL}$ in most curves, which increase as the reaction proceeds. The chromatograms for the samples taken immediately after mixing the reactants $(<1$ min) seem to have broad peaks, with relatively small peaks seen with a $20 \mathrm{~mL}$ retention volume. This suggests that the mPEG-SPA is quickly associated with the protein species and is then released relatively slowly. This conjecture is confirmed by the very broad elution profiles of this component during SERC runs (figures 5 and 6). 
Also a small peak containing low molecular weight components occurs in figures 3 and 4 at approximately the total column volume $(23.5 \mathrm{~mL})$ at the beginning of the reactions but is not present at later times. This peak has not been identified but is possibly the result of acidifying free activated PEG with $\mathrm{HCl}$.

$\mathrm{K}_{\mathrm{av}}$ values were calculated for the reaction products from their retention volumes on the analytical column. The standard curves in figure 1 show that proteins and PEG's behave differently in the size exclusion column. Using the PEG standard curve in figure 1 to determine molecular weights of PEGylated proteins from their $\mathrm{K}_{\mathrm{av}}$ values results in significantly underestimated molecular weights, although there is a linear relationship (correlation coefficient, $\mathrm{R}^{2}>0.99$ ) between the number of PEG groups attached and the molecular weight estimated by this means (figure 11). Each additional 5 kDa PEG group attached consistently adds $7 \mathrm{kDa}$ in effective molecular weight to the PEGylated product of both proteins. This, along with the step-wise changes in analytical size exclusion elution volumes for the reaction products, is good circumstantial evidence for concluding that the number of PEG groups attached to the products has been correctly identified.

Use of the protein standard curve in figure 1 significantly overestimated the molecular weight of the PEGylated proteins, with each additional PEG group adding between 50 and $100 \mathrm{kDa}$ (figure 11) to the apparent molecular weight. In contrast to the use of the PEG standard curve, the relationship between the number of PEG groups added and the increase in apparent molecular weight is not linear. 
Despite the consistent $7 \mathrm{kDa}$ increase in apparent molecular weight with each PEG group added to both proteins, it is clear that the actual molecular weights of the PEGylated proteins are not correctly estimated using PEG standards. The actual molecular weights of the PEGylated protein products identified by size exclusion were calculated from the known molecular weights of the native proteins and that of the activated PEG and these were then plotted in figure 1 against the $K_{a v}$ values calculated from their retention volumes. As might be expected, PEGylated proteins behave neither as proteins nor as PEG's with respect to their size exclusion behaviour. There is a consistent trend for the size exclusion behaviour of both proteins to move progressively from the protein standard curve towards the PEG standard curve with each additional PEG group attached.

Because PEG is transparent to UV light, it is reasonable to conclude that the UV absorbance of PEGylated species is due only to the protein part of each molecule and therefore an estimate of batch PEGylation reaction rates can be obtained from figures 3 and 4 . Figure 12 shows the change with time of the native protein peak areas as a percentage of the total protein peak areas in figures 3 and 4. Assuming the peak areas are directly proportional to the protein concentration, this indicates that $\alpha$-lactalbumin reacts faster than $\beta$-lactoglobulin, though the difference is not particularly large. The initial concentration of each reactant in the batch reactions was $10 \mathrm{mg} / \mathrm{mL}$. This corresponds to initial molar concentrations of $0.70,0.28$ and $2.0 \mu \mathrm{mol} / \mathrm{mL}$ of $\alpha$ lactalbumin, $\beta$-lactoglobulin and mPEG-SPA, respectively. However, it is not the total molar concentration of each protein but of each reactive site that is important. mPEG-SPA has one reactive site per molecule and this reacts with lysine groups and N-terminal amines. An examination of their 3D structures (Berman et al. 2000) shows 
that $\alpha$-lactalbumin has 4 lysine groups and the $\mathrm{N}$-terminal amine group accessible at its surface, while the $\beta$-lactoglobulin monomer has 5 lysine groups accessible at the surface. All other lysine groups on both proteins and the N-terminal amine group of the $\beta$-lactoglobulin monomer are at least partially "buried” within the molecule so can be assumed to be inaccessible for PEGylation. $\beta$-lactoglobulin exists as a dimer at the $\mathrm{pH}$ used in this study, so assuming that the accessible lysine groups of each monomer are not obscured by the formation of the dimer, we can estimate that $\beta$-lactoglobulin has 10 reactive sites per mole of dimer, while $\alpha$-lactalbumin has 5 per mole of monomer. The initial concentrations of active sites are therefore 3.5, 2.8 and 2.0 $\mu \mathrm{mol} / \mathrm{mL}$, for $\alpha$-lactalbumin, $\beta$-lactoglobulin and mPEG-SPA, respectively. Thus, at least to a first approximation, the faster reaction rate of $\alpha$-lactalbumin and the evidence of tri-PEGylated $\alpha$-lactalbumin in figure 3 and the absence of tri-PEGylated $\beta$-lactoglobulin in figure 4 are consistent with the initial reactant concentrations. In both cases the limiting reactant was mPEG-SPA. Other factors may come into play such as the mobility of the proteins' 3D structures and possible obscuration of nearby reactive sites by attached PEG groups. A more detailed analysis of the reaction kinetics is beyond the scope of this paper.

The broadness of the residual PEG molecule peak, its early appearance and its shape (figures 5 and 6), suggests that residual PEG was carried along the column with the larger molecular weight species, supporting the conjecture above that PEG quickly becomes associated with the protein species but is released more slowly. Virtually all protein was eluted (with little residual PEG content) from the column by the end of the major peak in both cases. It is unclear why these residual PEG molecules are eventually released rather than being covalently bonded to the protein. 
The difference in the molecular weight profiles between SERC (figures 9 and 10) and batch (figures 3 and 4) PEGylation is consistent with the hypothesis that the larger, PEGylated molecules are partitioned into the void volume and move out of the reaction zone at a higher rate than the native protein, thereby reducing the probability that they will be involved in further PEGylation.

Differences in the extent of reaction in SERC might be expected because of differences in the mobile phase partitioning of the two proteins i.e. $\beta$-lactoglobulin has a higher concentration in the void space than does $\alpha$-lactalbumin and therefore a greater proportion is available for reaction. The mobile phase partition coefficient, $\mathrm{K}_{\mathrm{d}}$, is given by

$$
K_{d}=\frac{V_{e}-V_{o}}{V_{i}}
$$

where $\mathrm{V}_{\mathrm{i}}$ is the volume of the mobile phase inside the gel which is available to very small molecules, $V_{e}$ is the elution volume of the molecule of interest and $V_{o}$ is the void volume of the size-exclusion column. $\mathrm{V}_{\mathrm{e}}$ was measured for each protein, $\mathrm{V}_{\mathrm{o}}$ was found from the retention volume of 2,000,000 molecular weight blue dextran (totally excluded from the gel pores), and $V_{i}$ was found from the difference in elution volume between the blue dextran and a sample of $\mathrm{NaCl}$ (the latter measured using a conductivity peak). $\mathrm{K}_{\mathrm{d}}$ values for $\alpha$-lactalbumin and $\beta$-lactoglobulin were calculated to be 0.4781 and 0.3085 , respectively. Using these $K_{d}$ values, the initial $10 \mathrm{mg} / \mathrm{mL} \alpha$ lactalbumin pulse is concentrated to $7.32 \mu \mathrm{mol} / \mathrm{mL}$ of active sites within the mobile phase of the SERC column, while $\beta$-lactoglobulin is concentrated to $9.08 \mu \mathrm{mol} / \mathrm{mL}$ of 
active sites. If there were no steric hindrance effects by the pores then one might expect $\beta$-lactoglobulin to react about $24 \%$ faster than $\alpha$-lactalbumin, based on the batch reaction results. Figure 9 and 10 show a greater extent of PEGylation of $\beta$ lactoglobulin than of $\alpha$-lactalbumin by SERC, although the extent of reaction would also be a function of the length of time the reactants overlapped in the column and therefore of their relative $\mathrm{K}_{\mathrm{av}}$ values.

It should also be noted that mPEG-SPA hydrolyses in water, with an expected halflife of about 30 to 45 minutes at the $\mathrm{pH}$ used in this study. A better choice of PEGylation reagent might be the more stable aldehyde or carbamate forms.

This suggests a method for controlling reactions so as to obtain a high yield of a product with a specific molecular weight. A size-exclusion medium that separates the reactants poorly but that sharply excludes products equal to or larger than the target PEGylated protein size would maximise the reaction time but minimise the formation of over-PEGylated products. This means that one could design a size-exclusion medium specifically for the production of, say, a di-PEGylated protein. The byproducts and residual PEG species, etc could be separated from the di-PEGylated protein product by collecting fractions in the exit stream, producing a largely homogeneous product at high yield. This, in combination with a reduction in capital costs and eliminating handling between reaction and separation steps, has the potential to greatly improve process economics.

The applicability of SERC is not restricted to protein PEGylation reactions but could potentially be used for any reaction that results in a change in molecular size, such as 
those involving glycosylation, polymerisation, or cleavage reactions, fusion proteins, poly- or oligo-peptides, amino acids, carbohydrates, inclusion bodies, microbial cells, viral particles, etc.

A number of useful avenues exist for further research. These include examining the effects of operating conditions on PEGylation reaction kinetics, the effect of pore size and pore-size distribution on the extent of reaction and the effect of the size-exclusion medium characteristics in combination with various PEGylation chemistries on PEGylation site specificity.

\section{References}

Abuchowski, A., McCoy, J.R., Palczuk, N.C., Van Es, T. and Davis, F.F. (1977). "Effect of covalent attachment of polyethylene glycol on immunogenicity and circulating life of bovine liver catalase.” Journal of Biological Chemistry 252(11): 3582-86.

APBiotech Gel filtration: Principles and methods. Uppsala, Sweden, Amersham Pharmacia Biotech.

Berman, H.M., Westbrook, J., Feng, Z., Gilliland, G., Bhat, T.N., Weissig, H., Shindyalov, I.N. and Bourne, P.E. (2000). “The protein data bank.” Nucleic Acids Research 28: 235-42.

Cunningham-Rundles, C., Zhuo, Z., Griffith, B. and Keenan, J. (1992). "Biological activities of polyethylene-glycol immunoglobulin conjugates. Resistance to enzymatic degradation.” Journal of Immunological Methods 152(2): 177-90. 
Fisher, D., Delgado, C., Selisko, B., Malik, F., Agrawal, A.K. and Francis, G.E. (1995). Covalent attachment of PEG to protein and liposomal surfaces. Biopolymer Mixtures. Harding, S.E., Hill, S.E. and Mitchell, J.R. Nottingham, Nottingham University Press. 56th: 421-35.

Francis, G.E., Fisher, D., Delgado, C., Malik, F., Gardiner, A. and Neale, D. (1998). "PEGylation of cytokines and other therapeutic proteins and peptides: The importance of biological optimization of coupling techniques.” International Journal of Hematology 68(1): 1-18.

Fung, W.-J., Porter, J.E. and Bailon, P. (1997). "Strategies for the preparation and characterization of polyethylene glycol (PEG) conjugated pharmaceutical proteins.” Polymer Preprints 38(1): 565-66.

Gombotz, W.R. and Pettit, D.K. (2000). PEGylation: A tool to enhance protein delivery. Controlled drug delivery: Designing technologies for the future. Park, K. and Mrsny, R.J. Washington, DC, American Chemical Society. 752: $110-23$.

\section{Acknowledgements}

Amersham Pharmacia Biotech provided size-exclusion columns and activated PEG reagent. The author is also grateful for useful comments and suggestions by Drs Lars Hagel and Lars-Erik Nystrom, of Amersham Pharmacia Biotech, Uppsala, Sweden. 


\section{Figures}

Figure 1. Elution profiles of native $\alpha$-lactalbumin, $\beta$-lactoglobulin and mPEG-SPA on a Superdex 200 HR 10/30 size-exclusion column.

Figure 2. Calibration curves for Superdex 200 HR 10/30 size-exclusion column for protein standards and PEG standards. Measured $\mathrm{K}_{\mathrm{av}}$ values are also shown for the products resulting from batch PEGylation of $\alpha$-lactalbumin and $\beta$ lactoglobulin, for which the corresponding molecular weights are calculated from the number of PEG groups assumed to have been attached to each native protein.

Figure 3. The effect of reaction time on the apparent molecular weight of $\alpha$ lactalbumin for batch PEGylation.

Figure 4. The effect of reaction time on the apparent molecular weight of $\beta$ lactoglobulin for batch PEGylation.

Figure 5. The elution profile for SERC PEGylation of $\alpha$-lactalbumin (UV absorbance at $280 \mathrm{~nm})$.

Figure 6. The elution profile for SERC PEGylation of $\beta$-lactoglobulin (UV absorbance at $280 \mathrm{~nm})$.

Figure 7. Molecular weight profiles of fractions collected during SERC PEGylation of $\alpha$-lactalbumin. Chromatograms are stacked to aid readability. Numbers refer to fractions in figure 5 .

Figure 8. Molecular weight profiles of fractions collected during SERC PEGylation of $\beta$-lactoglobulin. Chromatograms are stacked to aid readability. Numbers refer to fractions in figure 6.

Figure 9. Molecular weight profiles of pooled protein fractions collected during SERC PEGylation of $\alpha$-lactalbumin. 
Figure 10. Molecular weight profiles of pooled protein fractions collected during SERC PEGylation of $\beta$-lactoglobulin.

Figure 11. Dependence of the apparent protein molecular weight on the number of PEG groups attached.

Figure 12. Rate of reactions for batch PEGylation of $\alpha$-lactalbumin and $\beta$ lactoglobulin. 
Figure 1

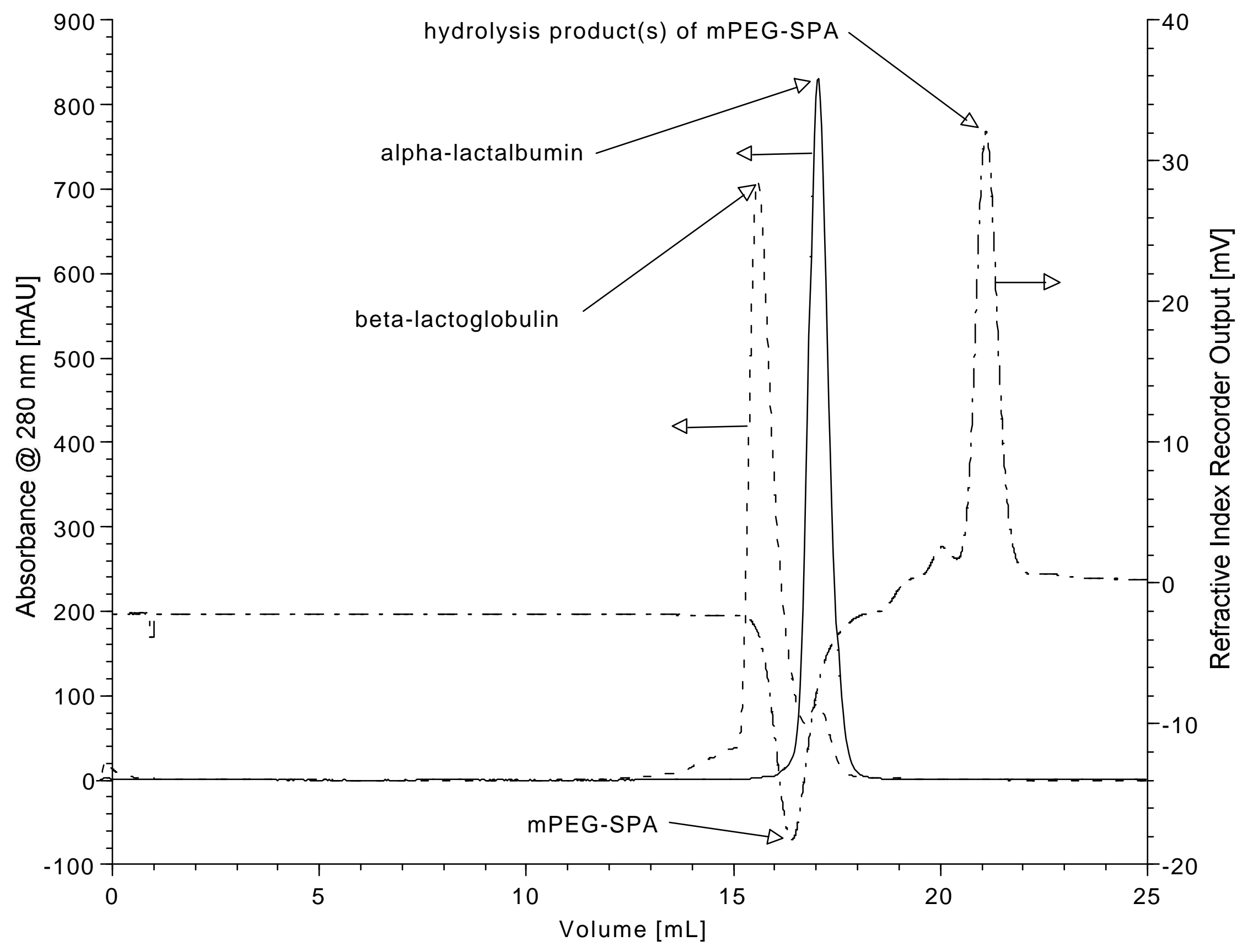


Figure 2

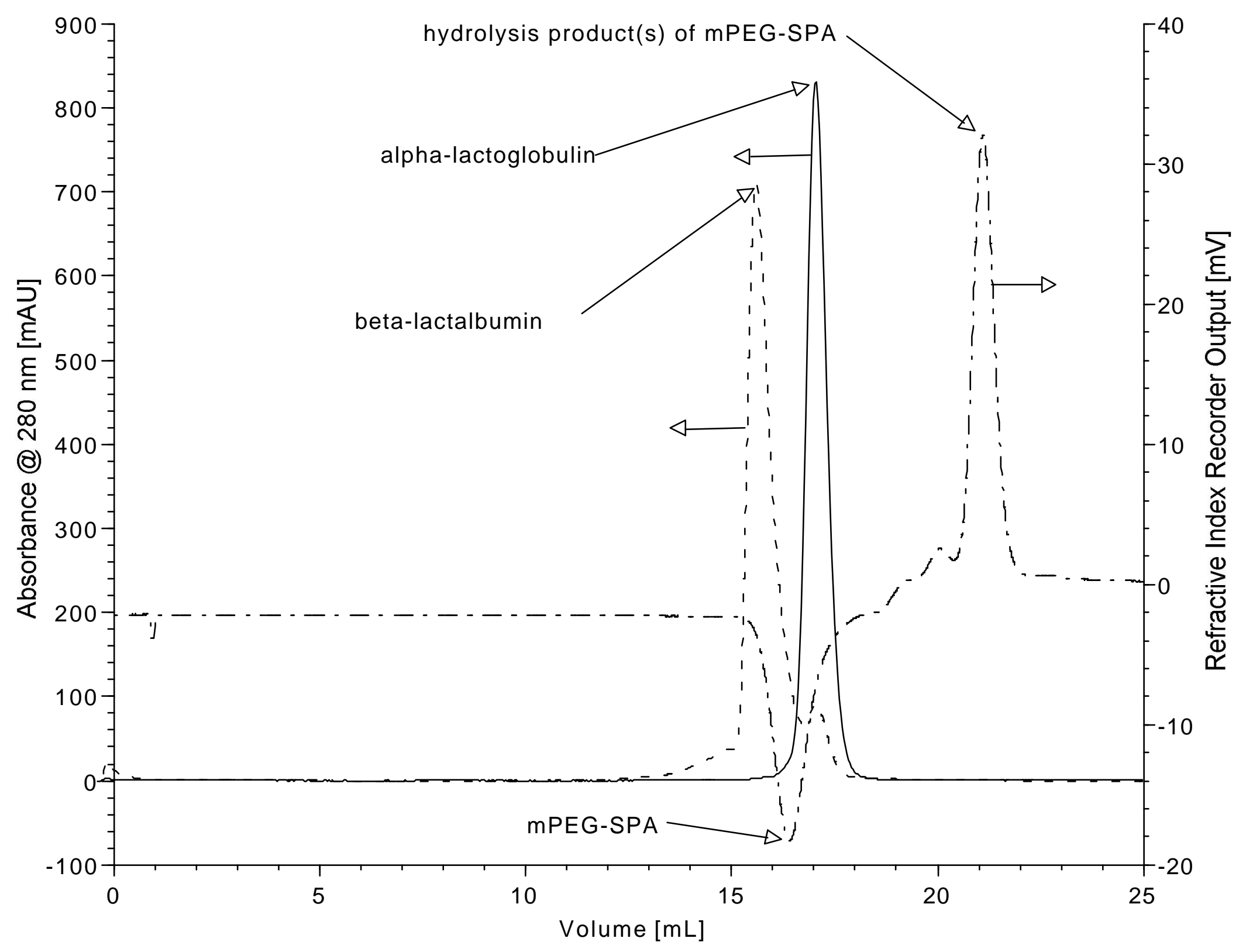


Figure 4

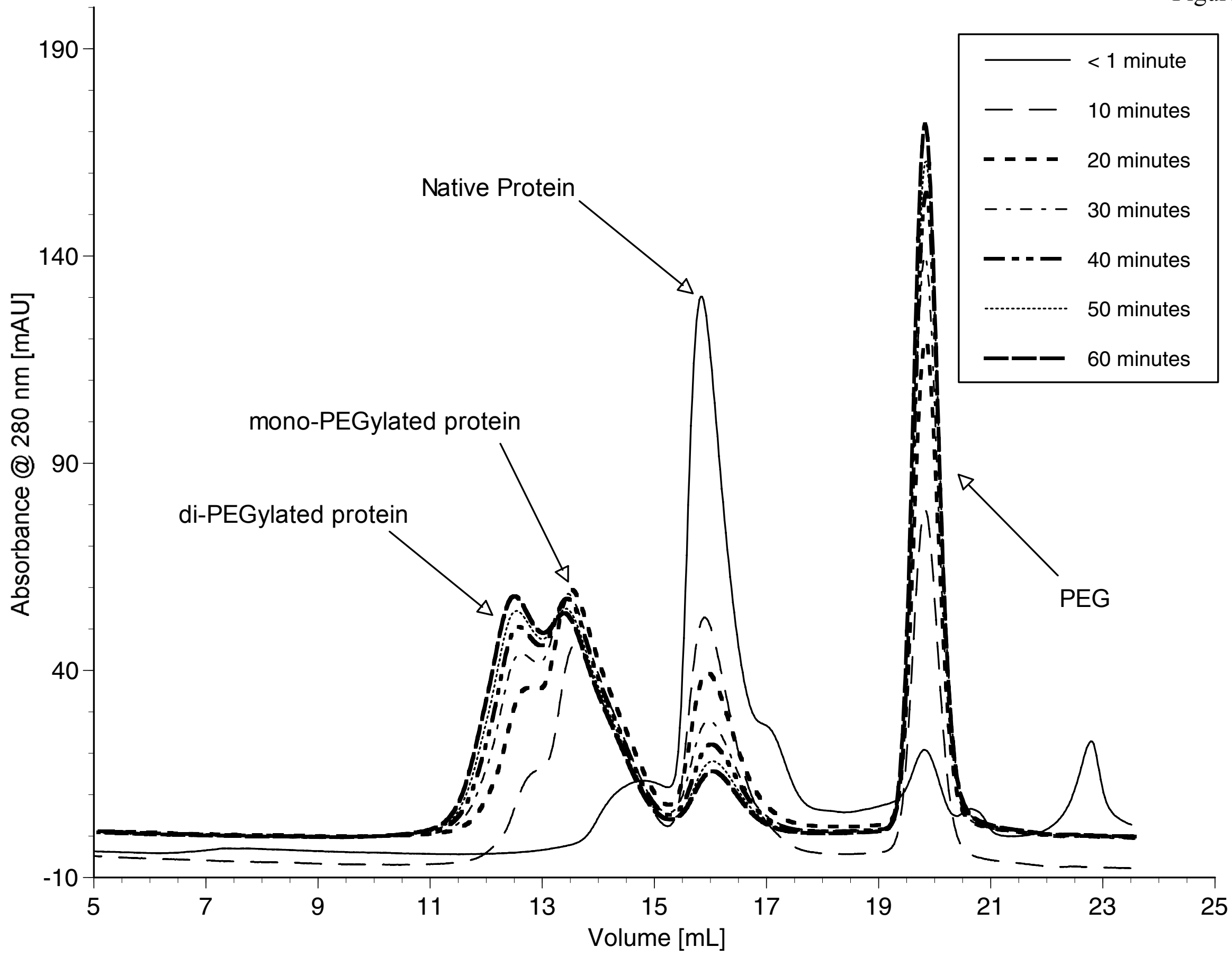


Figure 3

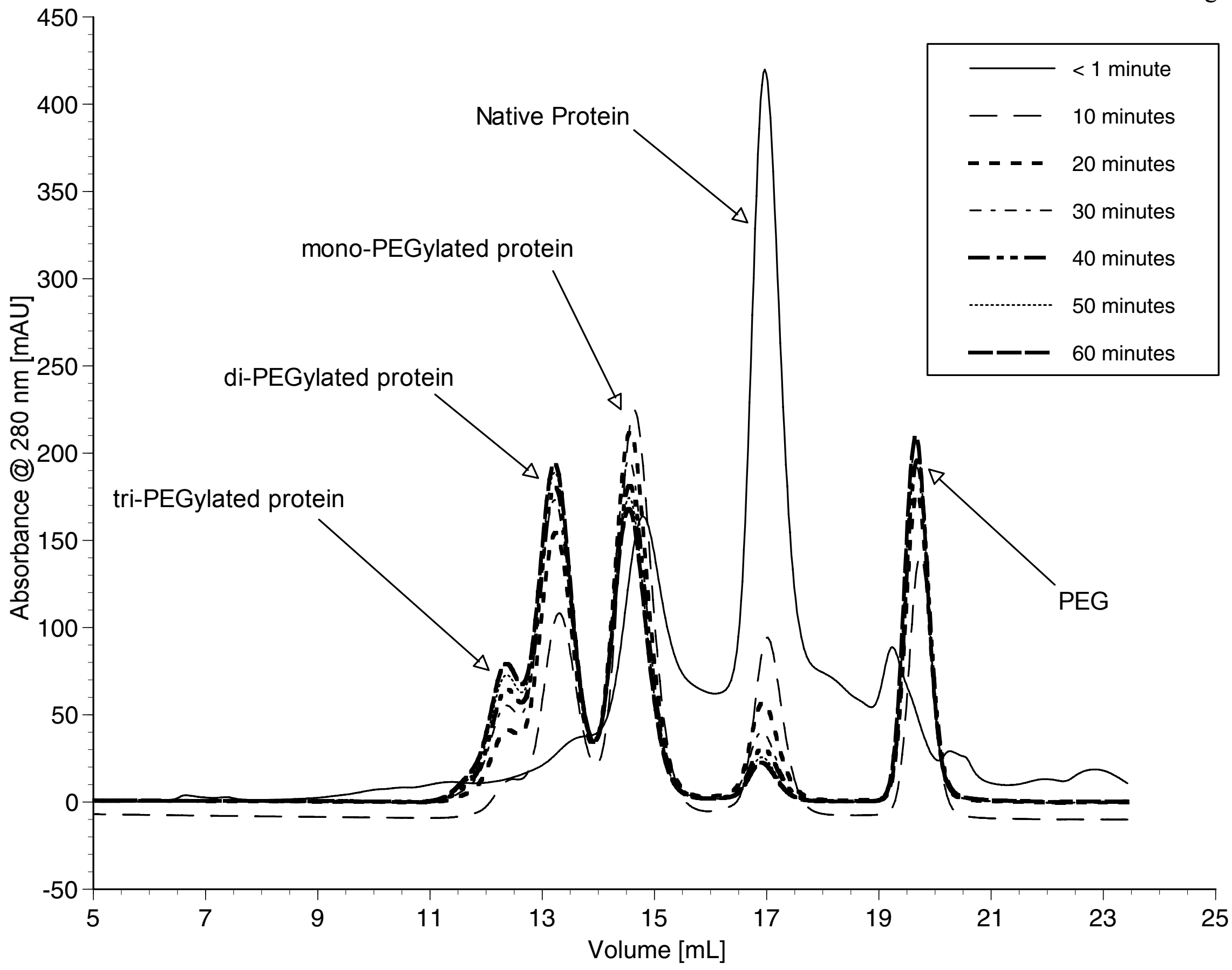


Figure 5

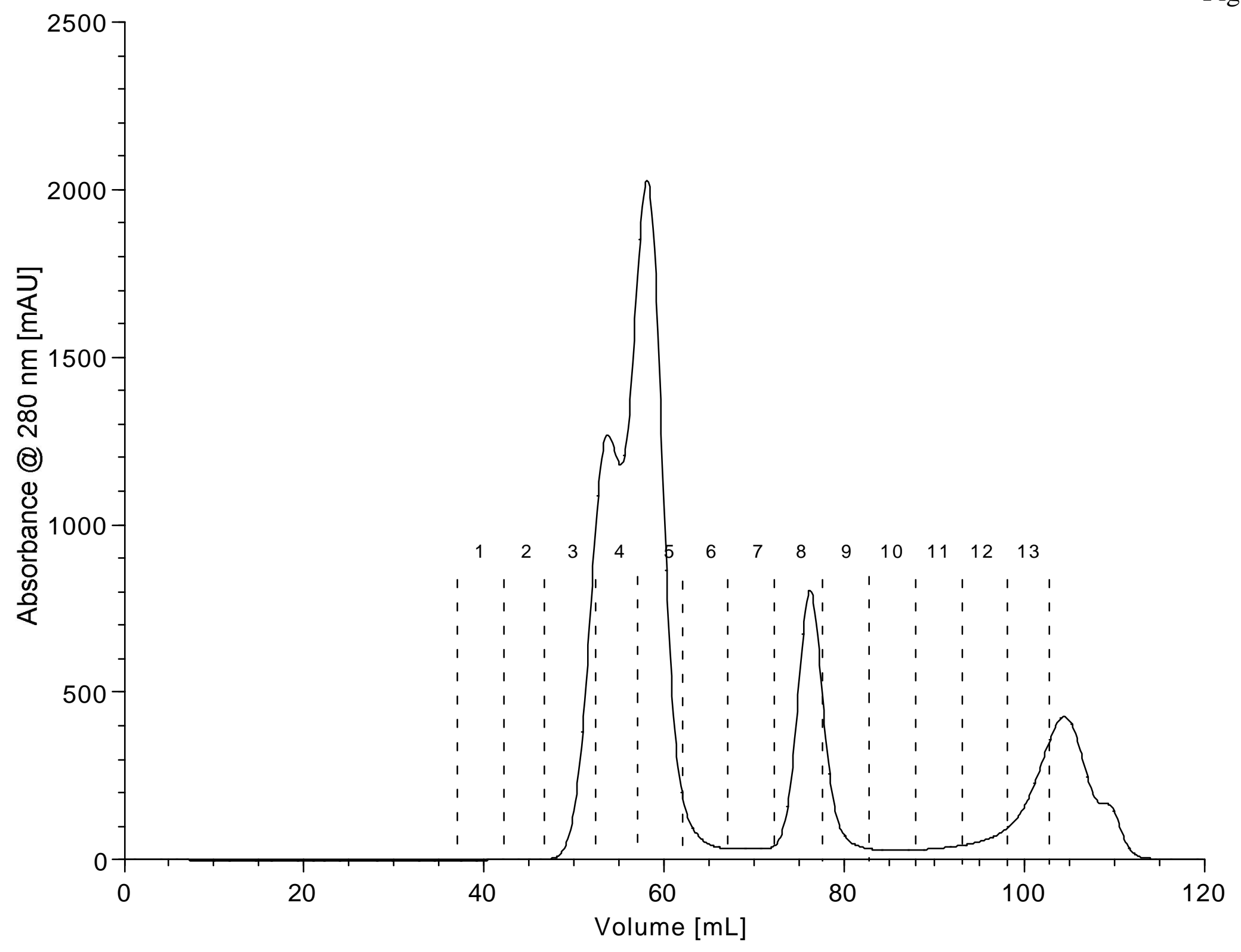


Figure 6

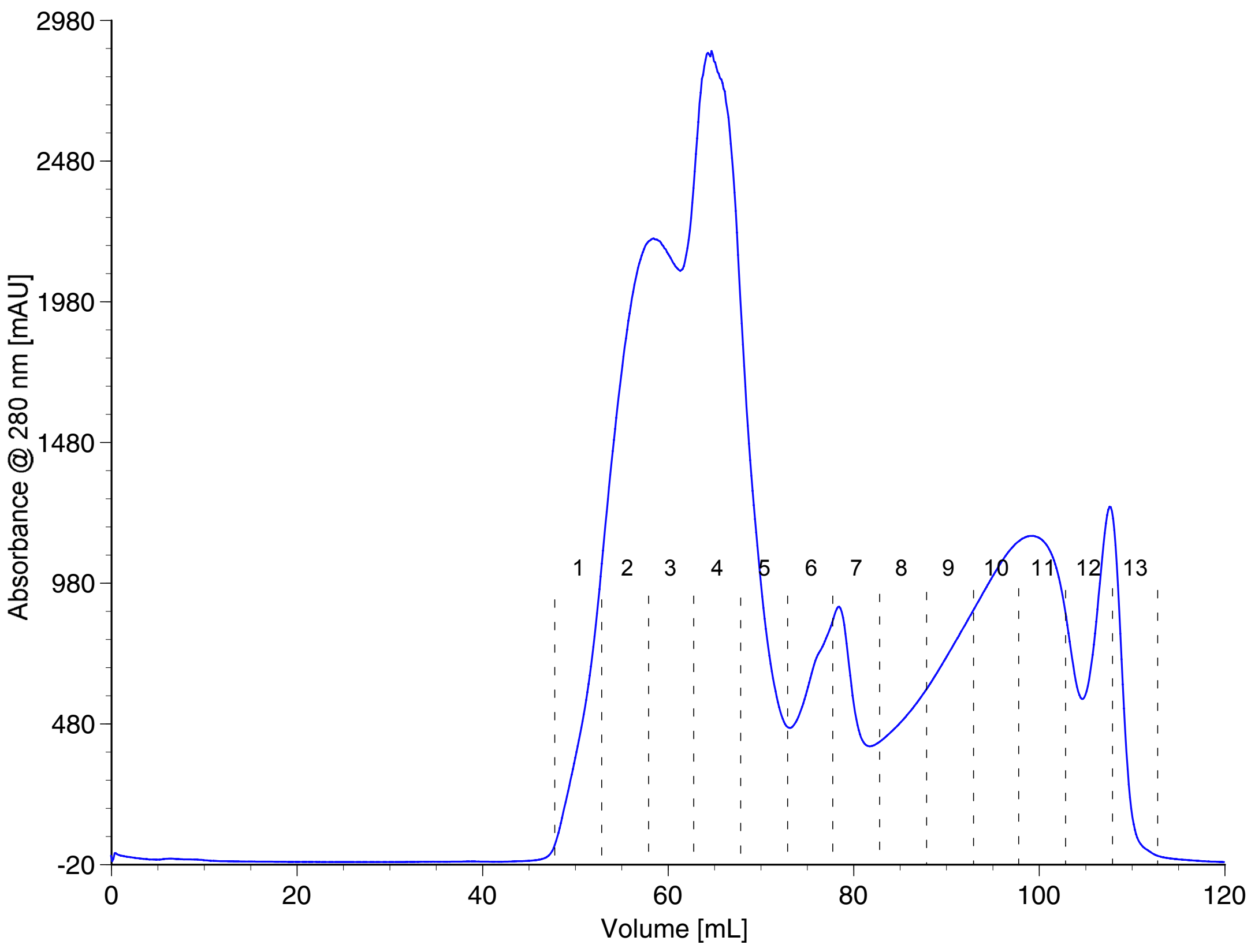


Figure 7

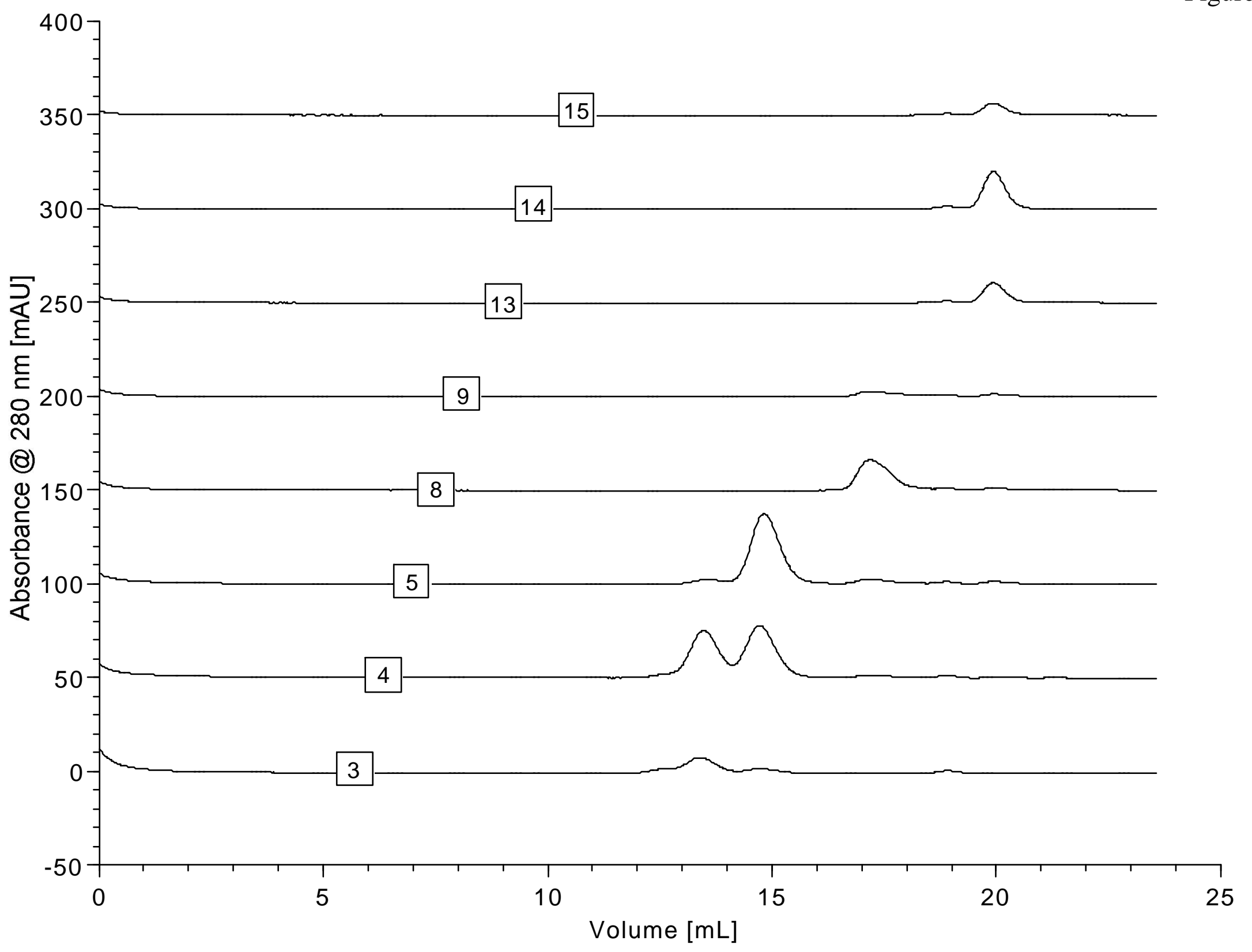


Figure 8

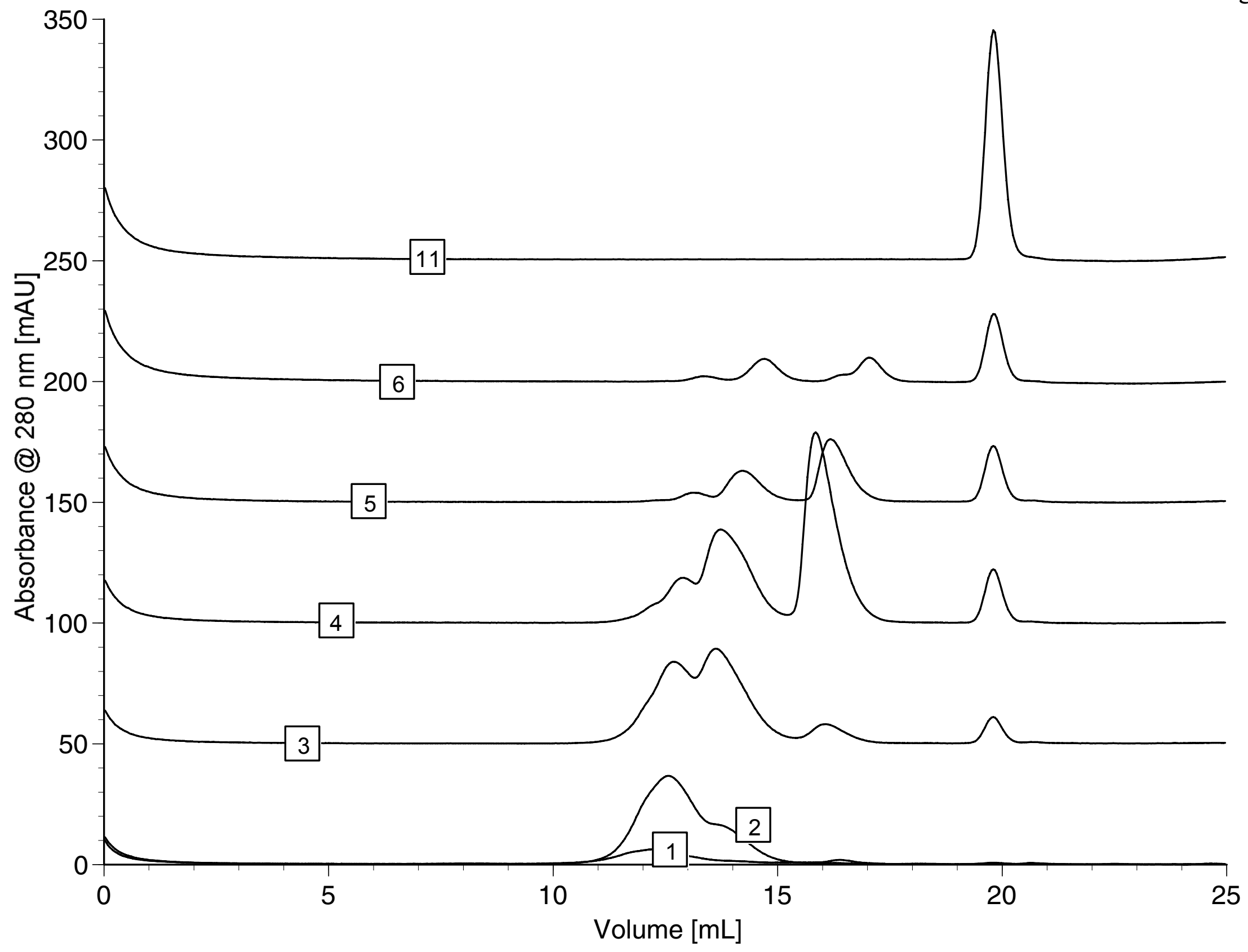


Figure 9

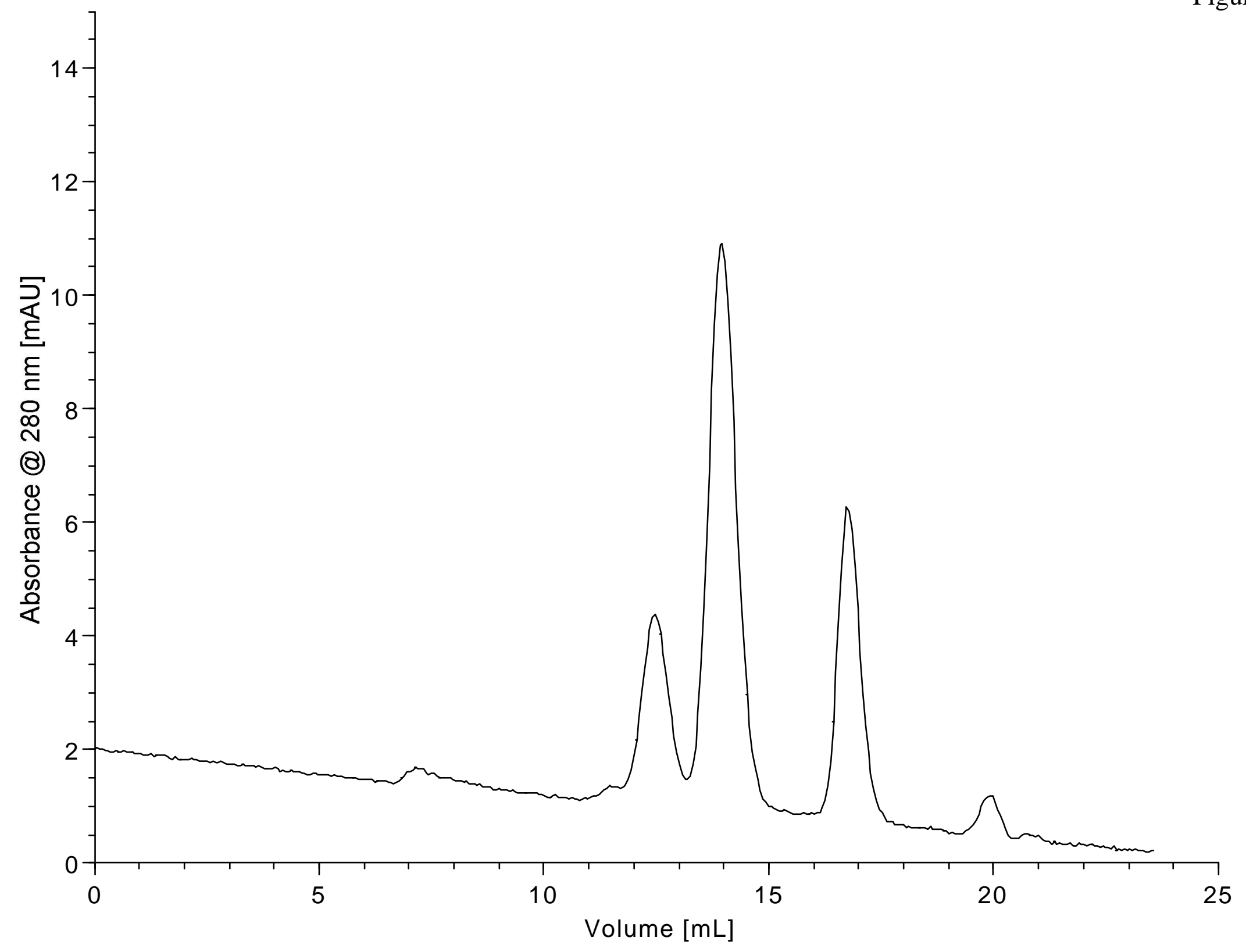




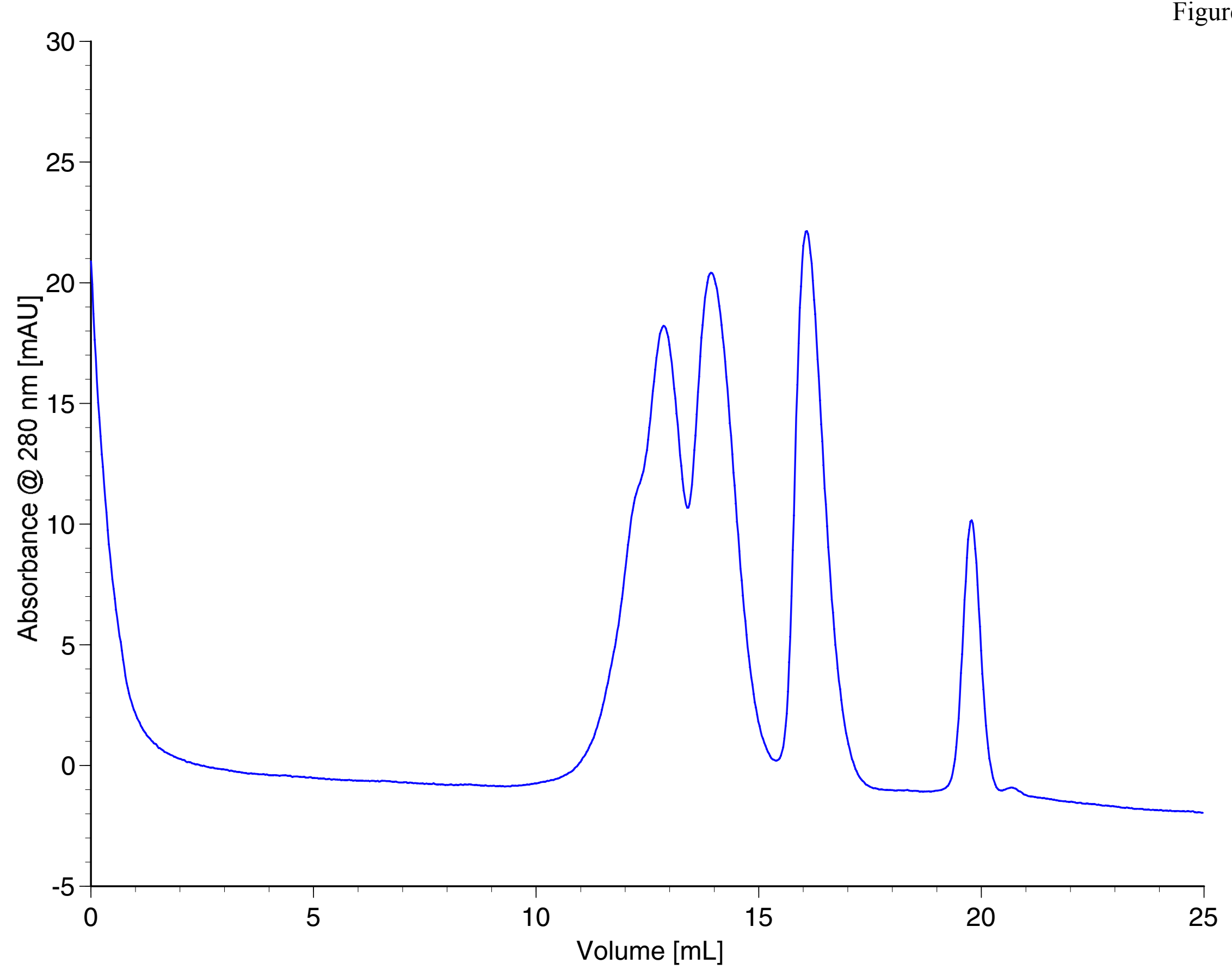


Figure 11

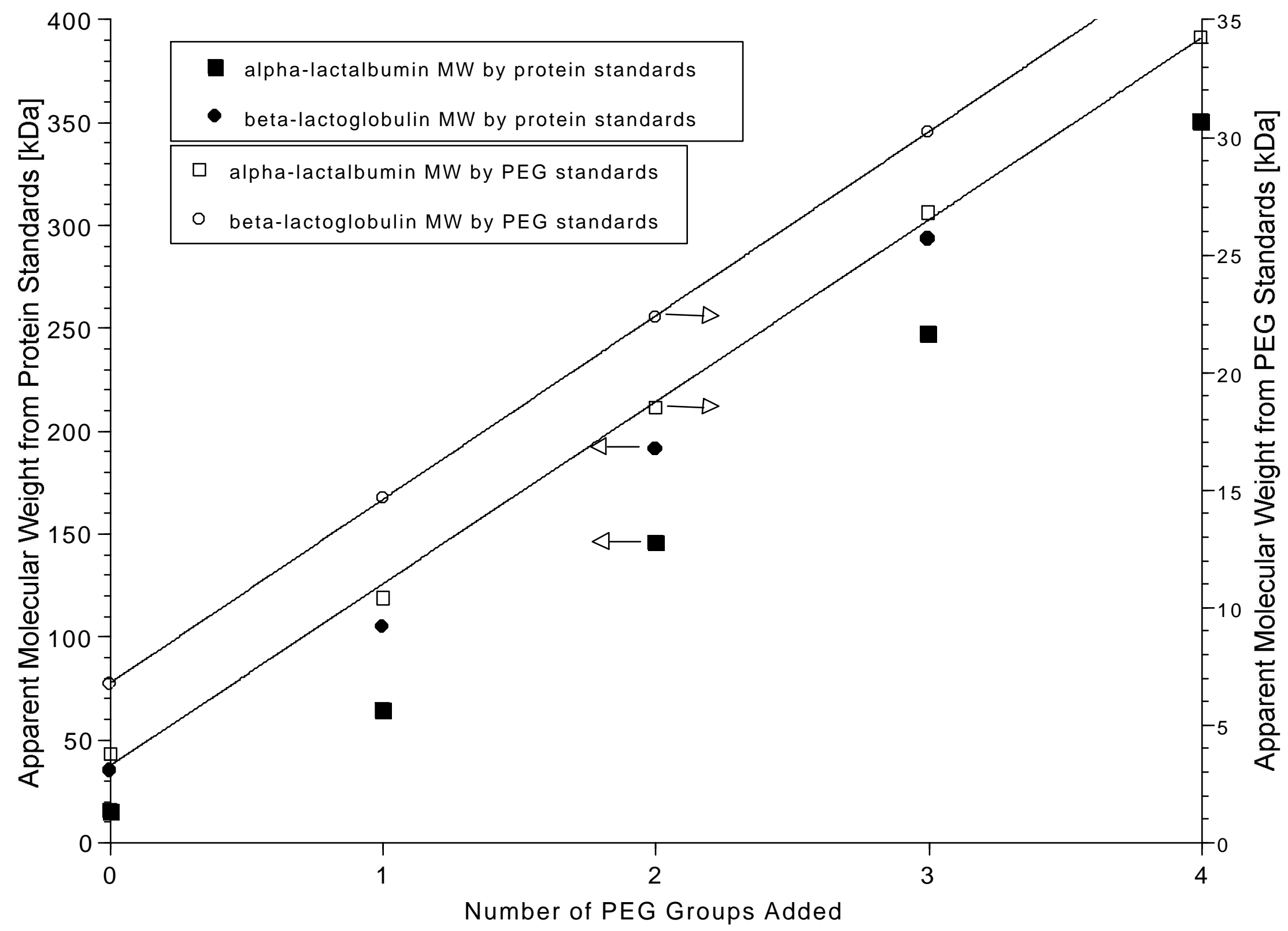


Figure 12

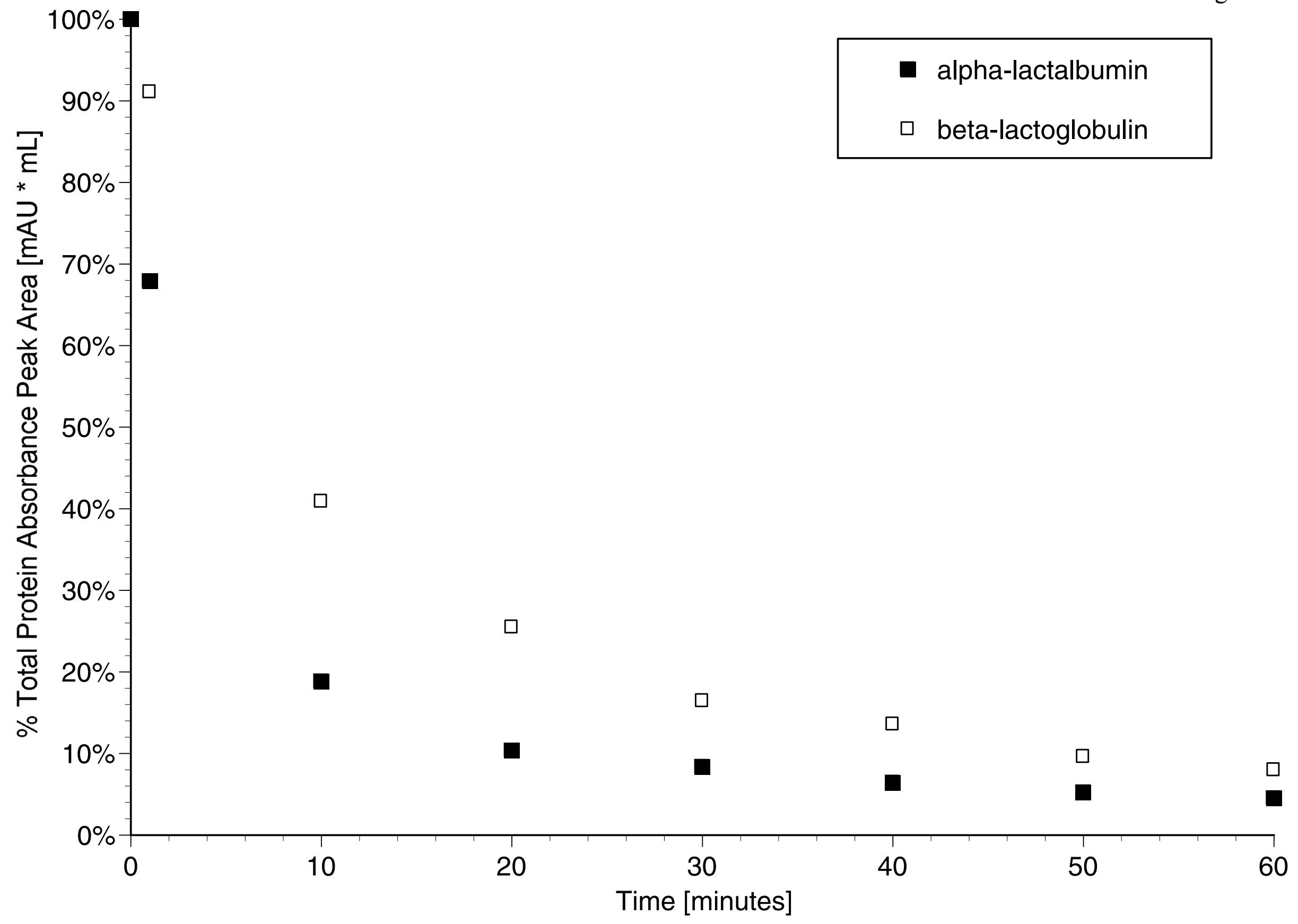

